\title{
COMMUNICATION
}

\section{Vescalagin and castalagin reduce the toxicity of amyloid-beta42 oligomers through the remodelling of its secondary structure}

Received 00th January 20xx, Accepted 00th January 20xx DOI: $10.1039 / x 0 \times x 00000 x$

\author{
Ana R. Araújo ${ }^{a, b, c}$, Sérgio Camero ${ }^{a, b, d}$, Pablo Taboadae, Rui L. Reis ${ }^{a, b, c}$ and Ricardo A. Pires ${ }^{a, b, c,{ }^{*}}$
}

The isomers vescalagin and castalagin protect SH-SY5Y cells from A 342 -mediated death. This is achieved better by vescalagin due to the spatial organization of its $\mathrm{OH}$ group at the $\mathrm{C} 1$ position of the glycosidic chain, improving its capacity to remodel the secondary structure of toxic $A \beta 42$ oligomers.

Alzheimer's Disease (AD) is the most common cause of dementia, characterized by cognitive impairment and memory loss. ${ }^{1}$ The most characteristic hallmarks of $A D$ are the presence of intracellular neurofibrillary tangles (of hyperphosphorylated Tau protein) in the affected neurons, and the deposition of extracellular plaques of amyloid- $\beta$ (A $\beta$ ) peptides in the hippocampus and entorhinal cortex. In the case of the amyloid deposits, they are usually composed of $A \beta$ of different lengths, i.e. between 38 and 43 amino acids. $A \beta$ is produced by neurons during the sequential proteolytic cleavage of amyloid precursor protein (APP). ${ }^{2} A \beta(1-42)(A \beta 42)$ is the less abundant species, however, it is the most amyloidogenic due to its higher propensity to self-assemble into supramolecular aggregates, which has been linked with the predominance of hydrophobic amino acid residues at its $\mathrm{C}$-terminus. ${ }^{3} \mathrm{~A} \beta 42$ can exist in several forms, e.g. monomers, oligomers or fibrils, however, its oligomeric species are reported to be the most cytotoxic ${ }^{4}$. They comprise different levels of association, such as dimers, trimers and higher hierarchical assemblies that lead to the formation of

\footnotetext{
a. 3B's Research Group, 13Bs - Research Institute on Biomaterials, Biodegradables and Biomimetics, University of Minho, Headquarters of the European Institute of Excellence on Tissue Engineering and Regenerative Medicine, AvePark, Parque de Ciência e Tecnologia, Zona Industrial da Gandra, 4805-017 Barco, Guimarães, Portugal.

b. ICVS/3B's-PT Government Associate Laboratory, Braga/Guimarães, Portugal.

The Discoveries Centre for Regenerative and Precision Medicine, Headquarters at University of Minho, Avepark, 4805-017 Barco, Guimarães, Portugal.

d. Present address: Institute of Physical Chemistry "Rocasolano", Spanish Nationa Research Council, Serrano 119, 28006 Madrid, Spain.

e. Colloids and Polymers Physics Group, Department of Condensed Matter Physics, Faculty of Physics, University of Santiago de Compostela, Campus Vida, E-15782 Santiago de Compostela, Spain.

"Corresponding author: rpires@i3bs.uminho.pt

Electronic Supplementary Information (ESI) available: [details of any supplementary information available should be included here]. See DOI: 10.1039/x0xx00000x
}

protofibrils, the intermediates of the $A \beta$ fibril formation and elongation. ${ }^{5,}{ }^{6}$ It has been also reported that these oligomeric structures spread out through the brain and trigger the hyperphosphorylation of Tau leading to the formation of the neurofibrillary tangles. ${ }^{7,} 8$ Despite the strong body of evidence that supports the pivotal role of oligomers on $A \beta$ toxicity, there is no agreement in the literature on their supramolecular organization. While some authors report them as being unstructured $^{9}$, others show that they present an anti-parallel $\beta$ sheet structure (in contrast to the parallel organization of the fibrils). ${ }^{10}$

Polyphenolic compounds are able to interact with $A \beta 42^{11-14}$. This interaction is mainly driven by: the binding of the polyphenols' aromatic rings with the amino and sulfhydryl groups of the peptide; or directly through $\pi-\pi$ stacking between the same aromatic rings and the $A \beta 42$ aromatic aminoacidic residues (e.g. phenylalanine). ${ }^{15}$ Based on these studies we evaluated the capacity of two cork polyphenols, i.e. vescalagin (1) and castalagin (2) (chemical structures presented in Fig. 1) to remodel the supramolecular organization of oligomeric $A \beta 42$ into non-cytotoxic forms. The extraction and identification of each compound was optimized and performed following a previous work $^{16}$ (chemical characterization is presented in Figs. S1-S7).

We started by evaluating the capacity of $\mathbf{1}$ and $\mathbf{2}$ to reduce the fibrillization of $A \beta 42$ using the Thioflavin-T (ThT) assay. ThT binds to $\beta$-sheet-rich structures mainly through $\pi-\pi$ interactions and displays enhanced fluorescence in the presence of amyloid fibrils. $^{17,} 18$ Initial ThT fluorescence was monitored for $\mathrm{A} \beta 42$ alone, showing the characteristic sigmoidal curve of peptide fibrillization (Figs. 2A and S12). To guarantee that there is no interference from the fluorescence of $\mathbf{1}$ and $\mathbf{2}$ in the results, their spectra were acquired in the absence of $A \beta 42$ (Figs. S8 and S9). At the excitation/emission wavelengths used for the ThT analysis, no fluorescence was observed for $\mathbf{1}$ and $\mathbf{2}$ showing that the ThT data is devoid of interference from the polyphenols themselves. After guaranteeing that the experimental conditions were set to provide meaningful data, $A \beta 42(25 \mu \mathrm{M}$ in 


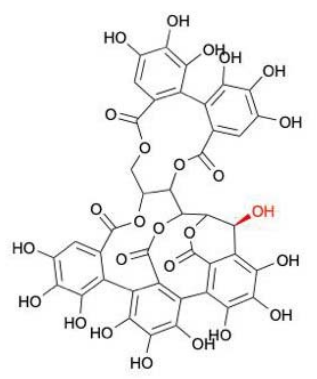

Vescalagin (1)

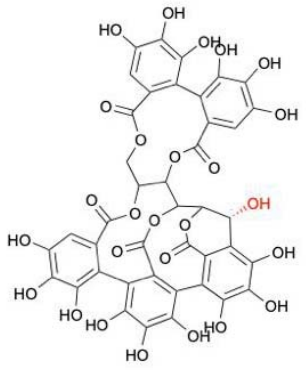

Castalagin (2)
Fig. 1 Chemical structure of the polyphenols vescalagin (1) and castalagin (2) extracted and purified from cork powder.

the monomeric form) was incubated at room temperature with ThT $(25 \mu \mathrm{M})$ and its fluorescence associated with the $A \beta 42$ secondary structure in the presence of 1 or $\mathbf{2}$ ( $A \beta 42$ :polyphenol molar ratios of $1: 0.5 ; 1: 1$ and $1: 2$ ) was followed over 140h (apx. 6 days). From Figs. $2 A$ and S13, it is clear the reduction of $A \beta 42$ fibrillization in the presence of $\mathbf{1}$ or $\mathbf{2}$ in a concentration dependent manner, where $\mathbf{1}$ was able to completely inhibit aggregation at an $A \beta 42$ :polyphenol ratio of 1:1, while 2 could only achieve this outcome at a ratio of 1:2.

We also tested the capacity of $\mathbf{1}$ and $\mathbf{2}$ to disassemble the A 342 pre-formed fibrils (Figs. 2B and S14). In this case, $\mathbf{1}$ and $\mathbf{2}$ were added at the plateau phase (after apx. 6 days of peptide incubation). The ThT fluorescence decreased immediately after the addition of the polyphenols ( $t=0-24 \mathrm{~h}$ ). Incubation of fibrils with an equimolar concentration of 1 reduced markedly the ThT fluorescence to apx. $20 \%$ at $\mathrm{t} \sim 20 \mathrm{~min}$ and to apx. $3 \%$ after $1 \mathrm{~h}$ (Fig. S14A). An almost coincident curve was recorded when a higher ratio of 1 was used (i.e. 1:2). In the case of 2 the ThT fluorescence reduced to apx. $50 \%$ at $\mathrm{t} \sim 20 \mathrm{~min}$ and to apx. $20 \%$ after $1 \mathrm{~h}$ (Fig. S14B). In both cases, assembly and disassembly, 1 seems to be more efficient in disrupting the $\beta$-sheet rich supramolecular assemblies of $A \beta 42$. Despite the reduction on ThT fluorescence, this method is not completely reliable to guarantee that no cytotoxic assemblies are formed. In fact, there are some reports in the literature that show that oligomer-specific antiparallel $\beta$-sheets do not exhibit ThT fluorescence, showing that this technique should not be considered on its own to evaluate the remodelling of $A \beta 42$ into non-cytotoxic forms. Considering these methodological constraints, we needed a complementary technique to ensure that we are indeed reducing the presence of $A \beta 42$ oligomers. We then evaluated by Western Blot (WB) if there was an observable reduction of the size of the peptide aggregates in the presence of 1 and 2 both during $A \beta 42$ assembly and disassembly (Figs. 2C-D and S15-S19). Overall, during assembly (i.e. 7 days of incubation, Fig. $2 \mathrm{C}$ and S15-S16) there is a clear reduction of $A \beta 42$ oligomers (between 2-3 peptide units, i.e. 15$10 \mathrm{kDa}$ ) from $\sim 35 \%$ to $\sim 18 \%$ in the presence of 1 . In contrast, in the presence of $\mathbf{2}$, only at the highest ratio $1: 2$, it starts to be observed a reduction of the oligomeric species, to $\sim 27 \%$. Importantly, we also observe a significant loss of larger $A \beta 42$ species (of different sizes: $250-55 \mathrm{kDa}$ ) for all the tested ratios (Fig. 2C). In the case of the $A \beta 42$ disassembly (Figs. 2D and S17-
S18) the presence of $\mathbf{2}$ seems to be more effective in in the

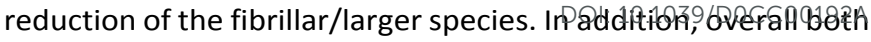
during assembly and disassembly, it is always detected a reduction of the $A \beta 42$ species detected by the $6 E 10$ monoclonal antibody. These results are consistent with a remodelling of the supramolecular organization of $A \beta 42$ into structures that are not able to be detected by immunostaining with 6 E10 (reported to detect different types of supramolecular A $\beta 42$ assemblies). ${ }^{5}$ Possible explanations are related with the $6 \mathrm{E} 10$ binding sites that, either are being occupied by the polyphenols, either the remodelled aggregates do not expose them on their surface. In any case, it is clear that $\mathbf{1}$ and $\mathbf{2}$ are able to partially alter the $A \beta 42$ supramolecular assemblies into forms that are not detectable by ThT nor by the 6E10 antibody.

While ThT and WB assays confirmed the capacity of $\mathbf{1}$ and $\mathbf{2}$ to alter the aggregation pathway of $A \beta 42$, it is important to look for the structural features that guide the observed remodelling. We then used circular dichroism (CD) to confirm if the peptide secondary structure is actually affected by the presence of $\mathbf{1}$ and 2. After 7 days of incubation, we observed that $A \beta 42$ alone presents a characteristic negative peak, at apx. $218 \mathrm{~nm}$ and a positive peak at $\sim 197 \mathrm{~nm}$ (Fig. S20). This spectral profile has been previously reported for the $\beta$-sheet conformation of oligomers. ${ }^{19,} 20$ Surprisingly, the addition of $\mathbf{1}$ or $\mathbf{2}$ (at the different ratios) does not change the overall spectral profile. However, both peaks present a blue shift after the addition of $\mathbf{1}$ or $\mathbf{2}$, showing that they promote an alteration of the $\beta$-sheet conformation of the $A \beta 42$ supramolecular assemblies (Fig. S20).

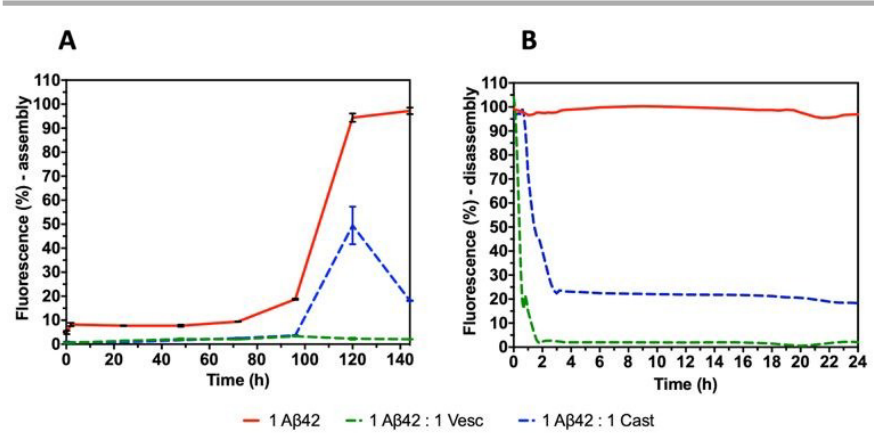

C D
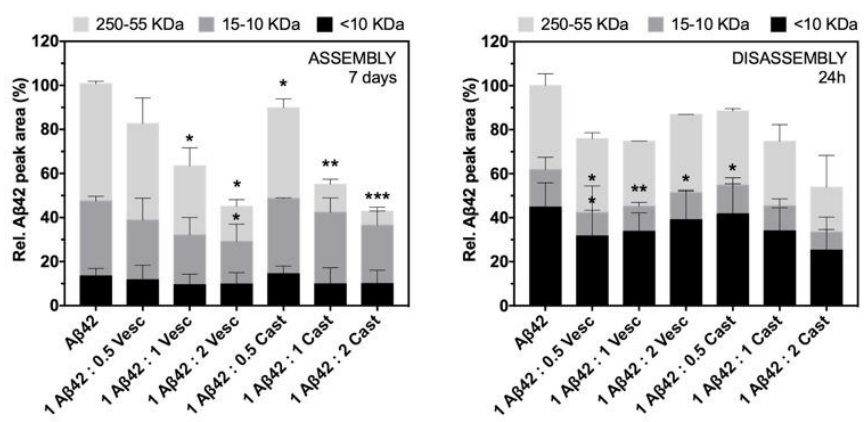

Fig. 2 A. Aggregation of $A \beta 42(25 \mu \mathrm{M})$ followed by ThT fluorescence upon incubation over $140 \mathrm{~h}$ under different $A \beta 42$ :polyphenol molar ratios, i.e. 1:0.5, 1:1 and 1:2. B. Disassembly of the fibrillar A $42(25 \mu \mathrm{M})$ followed by ThT fluorescence using the same $A \beta 42$ :polyphenol ratios. All measurements were recorded under constant agitation at $37^{\circ} \mathrm{C}$ and were monitored during $24 \mathrm{~h}$. Error bars $=\mathrm{SD} ; \mathrm{n}=3$. C-D. Relative densitometric bar graphs of C. A $342(25 \mu \mathrm{M})$ assembly and D. AB42 $(25 \mu \mathrm{M})$ disassembly quantified by WB (using the antibody 6E10); experiments conducted at $37^{\circ} \mathrm{C}$ in phosphate buffer, during C. 7 days or D. 24h; Error bars $=S D$, ${ }^{*} p<0.05, * * p<0.01$ and $* * * p<0.001$ vs control $25 \mu \mathrm{M} \mathrm{A \beta 42;} \mathrm{n}=3$. 


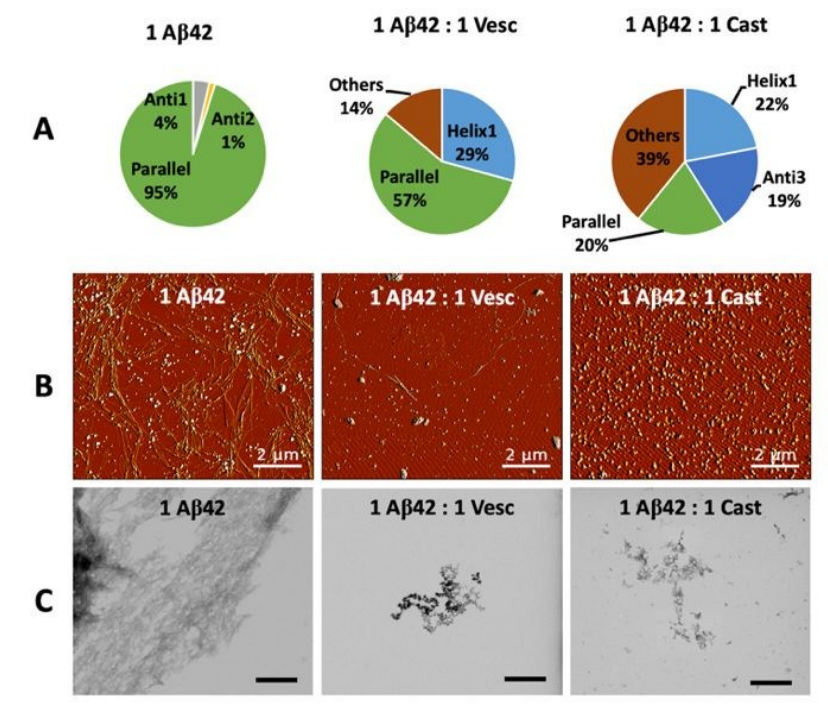

Fig. 3 A. Loss of parallel $\beta$-sheets by $A \beta 42(25 \mu \mathrm{M})$ monitored by $C D$ upon incubation with 1 and 2 under constant agitation at $37^{\circ} \mathrm{C}$, during $24 \mathrm{~h}$. $\mathrm{CD}$ data

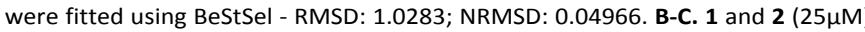
induced the refolding of $A \beta 42(25 \mu \mathrm{M})$ into spherical assemblies detected by AFM and STEM, respectively. Scale bars $2 \mu \mathrm{m}$ (AFM) and 500nm (STEM).

To evaluate if this shift was concomitant with a redistribution of the different types of the peptide secondary structure we fitted the CD spectra with BeStSel method for secondary structure estimation (Table S1). ${ }^{21}$ The results showed that $A \beta 42$ fibrils during the disassembly experiments ( $24 \mathrm{~h})$ are mainly composed by parallel $\beta$-sheet assemblies (Fig. 3A). ${ }^{21}$ We also found that both 1 and 2 decrease the parallel $\beta$-sheets content and promote a rearrangement of the peptide into helix, anti-parallel $\beta$-sheets and other types of secondary structures. Importantly, only $\mathbf{1}$ is able to eliminate the parallel arrangement without any anti-parallel organization. The remodelling of $A \beta 42$ promoted by 2 leads to the appearance of anti-parallel $\beta$-sheets. In the case of the assembly experiments, the $C D$ quantification (Table $\mathrm{S} 1$ ) is consistent with the ThT and WB analysis (Figs. 2A and 2C), showing a higher capacity of $\mathbf{1}$ to reduce the $\beta$-sheet content of Aß42, when compared to 2 .

The morphology of the generated assemblies was also monitored by AFM and STEM. Both $\mathbf{1}$ and $\mathbf{2}$ are highly efficient in remodelling the $A B 42$ fibrils (Figs. 3B-C and S22-S23). Both during assembly (24h) and disassembly (7days) experiments peptide aggregates were detected instead of long fibrils. Of note is that the oligomers observed in the $A \beta 42$ control, i.e. in the form of 10-15nm aggregates disappear in the presence of 1 or 2, giving rise to non-fibrillar larger aggregates. AFM images (Fig. S22) also confirmed that the remodelling of the $A \beta 42$ morphology is concentration-dependent.

Finally, considering that the association of the exposed hydrophobic peptide domains are the main driving forces for the intramolecular interactions, we evaluated the peptideligand binding energies (for both $\mathbf{1}$ and $\mathbf{2}$ ) at the thermodynamic level using isothermal titration calorimetry (ITC) ${ }^{22}$ (Table 1 and Fig. S24). In both cases $\Delta G$ is negative, indicating a tendency for the interaction to occur and that the formed peptide:ligand complex is thermodynamically stable. Our results show negative values for the other two thermodynamic parameters,
Table 1 ITC analysis of the interaction between $A \beta 42$ and iew $\mathrm{A} \mathrm{A}_{2}$ clinaling

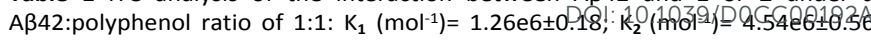
using a one binding site model.

\begin{tabular}{|c|c|c|c|}
\hline & $\begin{array}{c}\Delta H \\
\left(\mathrm{cal} \mathrm{mol}^{-1}\right)\end{array}$ & 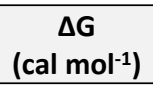 & $\begin{array}{c}\Delta S \\
\left(\text { cal mol } \mathrm{mol}^{-1} \mathrm{~K}^{-1}\right)\end{array}$ \\
\hline$A \beta 42: 1$ ratio $1: 1$ & $-9.28 e 4 \pm 0.91$ & $-11051 \pm 502$ & $-274 \pm 61$ \\
\hline $\mathrm{A} \beta 42: 2$ ratio $1: 1$ & $-4.58 \mathrm{e} 4 \pm 0.22$ & $-9082 \pm 290$ & $-123 \pm 45$ \\
\hline
\end{tabular}

i.e. $\Delta \mathrm{H}$ and $\Delta \mathrm{S}$. The enthalpic property, i.e. $\Delta \mathrm{H}$, is a measure of the average energy released along the interaction process between the peptide and the ligand. ${ }^{23}$ In this context, the negative value of $\Delta H$ (similarly to the results obtained for $\Delta G$ ) show that the formation of peptide:ligand complexes are energetically favourable; however, the negative values for $\Delta S$ indicate that the complexes are entropically unfavourable. This outcome can be explained by a progressive rupture of the hydration layer and release of counterions caused by the ring torsional degrees of freedom of each compound. In the case of $\mathbf{1}$ and $\mathbf{2}$, there is a covalent link between their aromatic rings generating a significant structural rigidity that is probably reflected in their interaction with $A \beta 42$. This rigidity might affect the hydrophobic interactions (e.g. $\pi-\pi$ stacking) promoting an increase on hydrogen bonding between the polyphenols and $A \beta 42$. In fact, $\mathbf{1}$ is reported to be more hydrophilic than $\mathbf{2}$ and with increased capacity to participate in hydrogen bonding due to the spatial organization of its $\mathrm{OH}$ at position $\mathrm{C} 1$ of the glycosidic chain. ${ }^{24}$ In addition, the interaction can also comprise charge shielding effects and/or molecular rearrangements of the ligand/peptide complexes. Interestingly, ITC data is consistent with the ThT, WB and CD results by suggesting that the complexes formed between compound 1 and $A \beta 42$ are more stable and energetically more favourable.

Our data suggests that both $\mathbf{1}$ and $\mathbf{2}$ are interacting with $\mathrm{A} \beta 42$, remodelling its supramolecular organization and morphological presentation, reducing the concentration of oligomers. To evaluate if compounds $\mathbf{1}$ and $\mathbf{2}$ are in fact able to reduce the $A \beta 42$-mediated cell death we performed a cytotoxicity assay using $\mathrm{SH}-\mathrm{SY} 5 Y$ cells. To this end, $\mathrm{A} \beta 42$ (in the monomeric form) was incubated with SH-SY5Y cells and their viability was quantified after $24 \mathrm{~h}$ using standardized MTS and Live/Dead assays. When A $\beta 42$ was added at a concentration of $25 \mu \mathrm{M}$, there was a decrease on cell viability to $\sim 50 \%$ (Fig. S25). Maintaining this concentration of $A \beta 42$, we added different concentrations of $\mathbf{1}$ and $\mathbf{2}$ (i.e. $12.5 \mu \mathrm{M}, 25 \mu \mathrm{M}$ and $50 \mu \mathrm{M}$ ). Cellular metabolic activity is recovered to $\sim 100 \%$, after $24 \mathrm{~h}$ (Fig. S29A) for all the tested conditions. In addition, Live/Dead assay (Figs. 4A) confirmed the same tendency showing that both 1 and 2 are able to rescue cell viability after $24 \mathrm{~h}$ of incubation. Quantification of the percentage of live cells (Fig. S28) revealed that the cytotoxicity of $A \beta 42$ resulted in less than $50 \%$ of live cells, however, $\mathbf{1}$ and $\mathbf{2}$ were able to rescue cells from death to a level similar to the control experiments, i.e. $\sim 100 \%$ of live cells. To understand if $A \beta 42$ was still present in the cellular space, we visualized the peptide by immunofluorescence (using the 6E10 monoclonal antibody, Figs. 4B and S30). The results clearly show a marked reduction in the fluorescence for $A \beta 42$ when 1 (but 
A
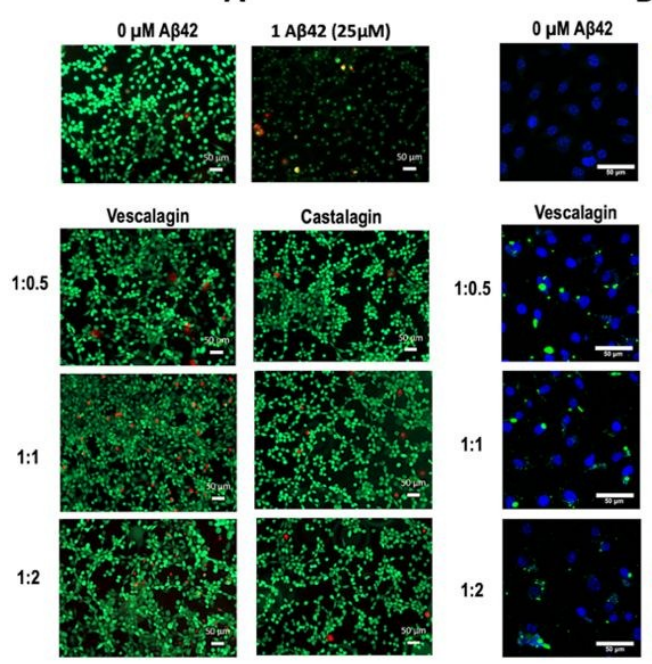

B
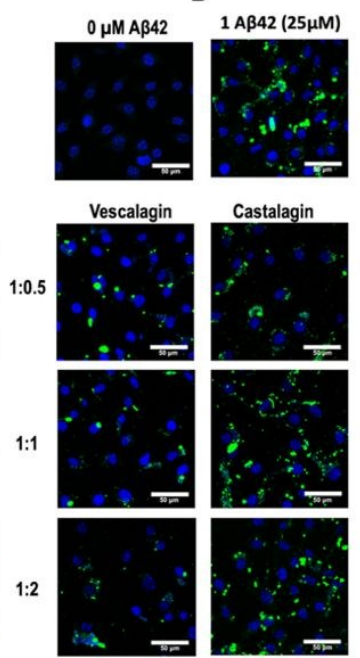

Fig. 4 A. Live/Dead assay using SH-SY5Y cells incubated with different $A \beta 42$ :polyphenol ratios during $24 \mathrm{~h}$ (green: live cells; red: dead cells). B. Immunofluorescence analysis of A 342 aggregates in the SH-SY5Y cell culture visualized by confocal microscopy (mAb 6E10, green) after incubation with 1 or for 24h (Aß42: green, cell nuclei: blue). Scale bar $=50 \mu \mathrm{m}$.

not $\mathbf{2}$ ) is added to the culture medium, showing that $\mathbf{1}$ is clearly more effective than 2 in reducing the presence of $A \beta 42$ aggregates in the cell culture. Of note, in the case of $\mathbf{1}$ (but not of 2) the reduction in fluorescence is also concentrationdependent, being able to reduce the fluorescence to $\sim 35 \%$ (Fig. S29B).

Overall, our data suggests that there is a higher propensity of $\mathbf{1}$ to interact with $A \beta 42$ when compared with $\mathbf{2}$. While being surprising, as the structural difference between $\mathbf{1}$ and $\mathbf{2}$ is only in the spatial organization of their $\mathrm{OH}$ group at the $\mathrm{C} 1$ position of the glycosidic chain, it has been already reported that this difference leads to higher reactivity, increased polarity and lower lipophilicity of $\mathbf{1}$ compared to $2 .{ }^{24}$ Overall, $\mathbf{1}$ is able not only to rearrange the $A \beta 42$ secondary structure, leading to the formation of non-cytotoxic structures (without their typical antiparallel $\beta$-sheets arrangement). Taken together, the ability of $\mathbf{1}$ to modulate the $A \beta 42$ aggregation pathway eliminating the characteristic cytotoxic oligomers is a step forward in the development of compounds with the capacity to control the trigger and/or progression of AD. Despite the higher activity of $\mathbf{1}$, it is clear that $\mathbf{2}$ is also able to interact with $A \beta 42$ and reduce its cytotoxicity towards the neuroblastoma cell line SH-SY5Y.

\section{Conflicts of interest}

The authors declare that there is no conflict of interest concerning the publication of this communication.

\section{Acknowledgements}

We acknowledge the financial support from the EC (FORECAST - 668983 and THE DISCOVERIES CTR - 739572), "Programa Operacional Regional do Norte", "Fundo Social Europeu", Norte2020 TERM\&SC, for the PhD grant NORTE-08-5369-FSE-000044; PT acknowledges Agencia Estatal de Investigación (AEI) for Project MAT2016-80266- $6_{e} R_{e}$ and "Xunta de Galicia" for grants "GPupd-10.19e9/LRefepeñảa Competitiva" ED431C 2018/26 and "Agrupación Estratégica en Materiales-AEMAT" ED431E 2018/08, respectively. ERDF funds are also acknowledged.

\section{References}

1. A. P. Merluzzi, C. M. Carlsson, S. C. Johnson, S. E. Schindler, S. Asthana, K. Blennow, H. Zetterberg and B. B. Bendlin, Neurology, 2018, 91, e436-e443.

2. D. J. Selkoe, Nature, 1999, 399, A23-A31.

3. L. Gremer, D. Scholzel, C. Schenk, E. Reinartz, J. Labahn, R. B. G. Ravelli, M. Tusche, C. Lopez-Iglesias, W. Hoyer, H. Heise, D. Willbold and G. F. Schroder, Science, 2017, 358, 116-119.

4. U. Sengupta, A. N. Nilson and R. Kayed, EBioMedicine, 2016, 6, 42-49.

5. R. Kayed, E. Head, F. Sarsoza, T. Saing, C. W. Cotman, M. Necula, L. Margol, J. Wu, L. Breydo, J. L. Thompson, S. Rasool, T. Gurlo, P. Butler and C. G. Glabe, Mol Neurodegener, 2007, 2, 18.

6. M. Necula, R. Kayed, S. Milton and C. G. Glabe, J Biol Chem, 2007, 282, 10311-10324.

7. S. Andrieu, N. Coley, S. Lovestone, P. S. Aisen and B. Vellas, Lancet Neurol, 2015, 14, 926-944.

8. J. Godyn, J. Jonczyk, D. Panek and B. Malawska, Pharmacol Rep, 2016, 68, 127-138.

9. D. E. Ehrnhoefer, J. Bieschke, A. Boeddrich, M. Herbst, L. Masino, R. Lurz, S. Engemann, A. Pastore and E. E. Wanker, Nat Struct Mol Biol, 2008, 15, 558-566.

10. D. L. Abeyawardhane, R. D. Fernandez, C. J. Murgas, D. R. Heitger, A. K. Forney, M. K. Crozier and H. R. Lucas, J Am Chem Soc, 2018, 140, 5028-5032.

11. P. Marambaud, H. Zhao and P. Davies, J Biol Chem, 2005, 280, 37377-37382.

12. C. Ramassamy, Eur J Pharmacol, 2006, 545, 51-64.

13. D. E. Ehrnhoefer, J. Bieschke, A. Boeddrich, M. Herbst, L. Masino, R. Lurz, S. Engemann, A. Pastore and E. E. Wanker, Nat Struct Mol Biol, 2008, 15, 558-566.

14. F. L. Palhano, J. Lee, N. P. Grimster and J. W. Kelly, J Am Chem Soc, 2013, 135, 7503-7510.

15. J. S. Wright, E. R. Johnson and G. A. DiLabio, J Am Chem Soc, 2001, 123, 1173-1183.

16. A. R. Araujo, D. M. Pereira, I. M. Aroso, T. Santos, M. T. Batista, M. T. Cerqueira, A. P. Marques, R. L. Reis and R. A. Pires, Rsc Adv, 2015, 5, 96151-96157.

17. Y. Porat, A. Abramowitz and E. Gazit, Chem Biol Drug Des, 2006, 67, 27-37.

18. J. T. Jarrett and P. T. Lansbury, Cell, 1993, 73, 1055-1058.

19. J. Bieschke, J. Russ, R. P. Friedrich, D. E. Ehrnhoefer, H. Wobst, K. Neugebauer and E. E. Wanker, Proc Natl Acad Sci U S A, 2010, 107, 7710-7715.

20. C. J. Barrow, A. Yasuda, P. T. Kenny and M. G. Zagorski, J Mol Biol, 1992, 225, 1075-1093.

21. A. Micsonai, F. Wien, L. Kernya, Y. H. Lee, Y. Goto, M. Refregiers and J. Kardos, Proc Natl Acad Sci U S A, 2015, 112, E3095-3103.

22. D. Chandler, Nature, 2005, 437, 640-647.

23. A. de Ruiter and C. Oostenbrink, Curr Opin Chem Biol, 2011, 15, 547-552.

24. N. Vivas, M. Laguerre, I. Pianet de Boissel, N. Vivas de Gaulejac and M. F. Nonier, Journal of agricultural and food chemistry, 2004, 52, 2073-2078. 